(0.93-1.47 mmol/l) and $\geq 131 \mathrm{mg} / \mathrm{dl}(\geq 1.48 \mathrm{mmol} / \mathrm{l})$, respectively on first measurement, and $\leq 93 \mathrm{mg} / \mathrm{dl}$ ( $\leq 1.05 \mathrm{mmol} / \mathrm{l}), 94-147 \mathrm{mg} / \mathrm{dl}$ (1.06$1.66 \mathrm{mmol} / \mathrm{l})$ and $\geq 148 \mathrm{mg} / \mathrm{dl}(\geq 1.67 \mathrm{mmol} / \mathrm{l})$ respectively on second measurement.

Within 5.5 years, 158 new cases of CHD were identified. Compared with men who had low triglyceride levels on both assessments, men with initially low levels became 3.81 times and 6.76 times more likely to develop CHD if their level rose to intermediate or high, and men with stable high triglyceride levels were over eight times more likely to develop CHD. Men with an initial high score that then reduced to intermediate or low were, respectively 6.84 times and 4.9 times more likely to develop CHD than were men with stable low levels. The changes in triglyceride levels were strongly correlated with lifestyle changes.

Two triglyceride measurements obtained 5 years apart could, therefore, augment the assessment of CHD risk in young men. The authors note that the risk of CHD in men whose triglyceride levels fluctuated from high to low remained elevated compared with men who had persistently low triglyceride levels.

Original article Tirosh A et al. (2007) Changes in triglyceride levels and risk for coronary heart disease in young men. Ann Intern Med 147: 377-385

\section{Study identifies risk factors associated with infection after device implantation}

Device-related infection is a serious complication of both cardiac pacing system and cardioverterdefibrillator implantation. The Prospective Evaluation of Pacemaker Lead Endocarditis (PEOPLE) study was set up to determine the incidence and risk factors associated with this complication. Klug et al. report the results of this trial in a recent paper published in Circulation.

The study population comprised 6,319 consecutive patients who underwent implantation of an antiarrhythmic system at one of 44 participating medical centers in France. In total, 4,465 de novo implantations and 1,854 pulse generator or lead replacements were carried out. Antibiotic prophylaxis was used in $88 \%$ of procedures. At 12 months, follow-up data were available for 5,404 (85.5\%) participants.

In total, 42 patients developed infectious complications over the 12-month study period-an incidence of device-related infection of 0.68 (95\% Cl 0.47-0.89) per 100 patients. The median time to infection was 52 days. The incidence of infection was higher in patients undergoing device replacement than it was in those undergoing de novo procedures (0.99 per 100 patients vs 0.56 per 100 patients). Multivariate analysis demonstrated positive associations between the incidence of device-related infection and the following factors: fever within the $24 \mathrm{~h}$ before implantation; the presence of a temporary pacing system at the time of implantation; and early reintervention for hematoma or lead replacement. The use of antibiotic prophylaxis and implantation of a de novo system were negatively related to the incidence of infection.

Original article Klug D et al. (2007) Risk factors related to infections of implanted pacemakers and cardioverterdefibrillators: results of a large prospective study. Circulation 116: $1349-1355$

\section{Outcomes of revascularization with MECC similar to those with off-pump CABG surgery}

The advantages that off-pump (OP)CABG surgery has over full cardiopulmonary bypass in terms of reduced morbidity are well documented, although revascularization with this technique can be difficult in individuals with complex lesions. Initial experience with the minimal extracorporeal circulation system (MECC) indicates that this method might also confer considerable postoperative benefits compared with full cardiopulmonary bypass. Now, Mazzei and colleagues have conducted a prospective trial to directly compare the early and 1-year outcomes of myocardial revascularization performed with the use of either OPCABG or MECC.

In total, 300 patients were randomized on a 1:1 basis to receive coronary surgery with OPCABG or MECC. The mean duration of coronary surgery with MECC was significantly longer than that of surgery with OPCABG $(P=0.027)$; however, 30-day mortality, duration of stay in intensive care, duration of hospitalization, the complications rate, and the need for allogeneic transfusion were similar in the two groups. The levels and patterns of release of interleukin 6 , creatine kinase and S-100 protein-markers of systemic inflammation, myocardial injury and brain injury, respectively - were also similar in the two groups. Mortality at 1 year was $2.7 \%$ 